\title{
Wahrgenommene Umsetzung des integrierten Pflanzenschutzes bei landwirtschaftlichen Betrieben in Nordwestdeutschland
}

\author{
Lukas Thiel $^{1}$ - Marcus Mergenthaler ${ }^{1}$ - Verena Haberlah-Korr ${ }^{1}$ \\ Eingegangen: 17. November 2020 / Angenommen: 21. Januar 2021 / Online publiziert: 10. Februar 2021 \\ (c) Der/die Autor(en) 2021
}

\section{Zusammenfassung}

Die acht allgemeinen Grundsätze des integrierten Pflanzenschutzes (IPS) sind als über die Kulturarten allgemeingültiger Leitfaden zur Umsetzung des IPS auf den landwirtschaftlichen Betrieben gedacht. Sie greifen vorbeugende und aktiv eingreifende Methoden zur Verminderung des Schädlingsdrucks an den Kulturpflanzen auf und zeichnen ein derzeitiges Idealbild des Pflanzenschutzes. Eine Betrachtung der Umsetzung in Deutschland hat bisher nicht stattgefunden und soll in dieser Studie exemplarisch für den nordwestdeutschen Raum erfolgen. Zugrunde liegen Daten einer Befragung durch die FH Südwestfalen aus dem Herbst 2019. Es zeigt sich, dass insbesondere Erfolgskontrollen, zielartenspezifischer Pflanzenschutzmitteleinsatz und Strategien der Resistenzvermeidung auf den Betrieben genannt werden. Feldkontrollen vor Pflanzenschutzmaßnahmen sowie der Einsatz nicht-chemischer Methoden gerade in der Beikrautregulierung werden hingegen noch nicht als umfangreich praktiziert angegeben. Gerade im Bereich der vorbeugenden Methoden und dem Einsatz von Schadschwellen fehlen standortspezifische, praxistaugliche Handreichungen. Dieses Fehlen hindert eine weitere Umsetzung der acht allgemeinen Grundsätze des integrierten Pflanzenschutzes. Es sind weiterhin regionalspezifische Forschungen und Tests notwendig, um diese Lücke zu schließen.

Schlüsselwörter Integrierter Pflanzenschutz · Umsetzungsstudie · Konventionelle Landwirtschaft · Befragung

\section{Perceived Implementation of Integrated Pest Management on Farms in North-West Germany}

\begin{abstract}
The eight general principles of integrated pest management (IPS) are intended as general guidelines for the implementation of the IPS on farms across any crop types. They take up preventive and actively intervening methods to reduce pest pressure on the cultivated plants and paint an ideal picture of crop protection. The implementation in Germany has not yet been analyzed. This study is intended to partly fill this research gap by considering north-west Germany as an example. It is based on data from a telephone survey done with 300 conventional farmers from the north west of Germany in autumn 2019.

It has been shown that success controls, target species-specific plant protection products and strategies for avoiding resistance on the farms are given particular attention. On the other hand, field controls before plant protection measures, as well as the use of non-chemical methods, especially in weed control, are not yet widely practiced. In the part of preventive methods and the use of damage thresholds, however, there is also a lack of location-specific, practical instructions. This absence prevents further implementation of the eight general principles of integrated pest management. Region-specific research and testing are still needed to reduce the implementation gap.
\end{abstract}

Keywords Integrated pest management $\cdot$ Documentation of realization $\cdot$ Conventional farming $\cdot$ Survey

Lukas Thiel

thiel.lukas@fh-swf.de

1 Fachbereich Agrarwirtschaft, Fachhochschule Südwestfalen, Soest, Deutschland

\section{Einleitung}

Der integrierte Pflanzenschutz steht seit den 80er-Jahren im Pflanzenschutzgesetz, wurde im Jahr 2010 in die ,gute fachliche Praxis“ integriert, 2014 gesetzlich festgeschrieben $(§ 3$ 
PflSchG 2012) und dient als Idealbild des Pflanzenschutzes (BMELV 2010; Müller 2001). 2015 stellte Hokkanen die These auf, dass die aktuelle Umsetzung des integrierten Pflanzenschutzes dem umgekehrten Idealbild entspricht und der chemische Pflanzenschutz die momentane Basis des Pflanzenschutzes und der Pflanzenschutzentscheidungen an Stelle ihrer letzten Option darstellt (Hokkanen 2015). Im Vergleich zu anderen Bestrebungen für mehr Umweltschutz im Pflanzenbau wird der IPS teilweise als gescheitert betrachtet (Haller et al. 2020).

Frühere Befragungen von 32 Betriebsleitern landwirtschaftlicher Betriebe aus Nordrhein-Westfalen in 2018/2019 zeigten, dass besonders arbeitsorganisatorische Aspekte, Zeit und teils geringe Kosten (hier: Insektizide) für Pflanzenschutzmittel ausschlaggebende Hemmnisse des IPS sind. Das wahrgenommene Risiko durch Schaderreger beeinflusst diese Haltung ebenfalls (Thiel et al. 2019).

Bisherige Forschungsansätze haben den integrierten Pflanzenschutz häufig auf Ebene von Kulturartengruppen - meist in Kombination mit Schädlingskategorien - betrachtet (Getreide und Pilzerkrankungen: Jorgensen et al. 2008a, b; Ungräser: Llewellyn et al. 2007). Ebenfalls wurde das Thema aus sozioökonomischer Sicht angegangen, beispielsweise, welche Faktoren Einfluss auf die Methodenadoption einwirken. Dazu wurde das Modell ADOPT entwickelt, welches über Fragen zur regionalen Struktur, den eigenen Voraussetzungen des Landwirts, aber auch objektive Vorteile neuer Methoden miteinander kombiniert. Daraus können Vorhersagen über mögliche Methodenadoptionen durch die Landwirte ermittelt werden (Kuehne et al. 2017). Eine Betrachtung bezogen auf die in der EU angewandten acht allgemeinen Grundsätze, insbesondere für die Anwendung des integrierten Pflanzenschutzes in Deutschland, liegt bisher nicht vor.

2009 wurden die acht allgemeinen Grundsätze des integrierten Pflanzenschutzes in der EU-Richtlinie 2009/128/EG Anhang III festgelegt (Richtlinie 2009/128/EG des europäischen Parlaments und des Rates vom 21. Oktober 2009 über einen Aktionsrahmen der Gemeinschaft für die nachhaltige Verwendung von Pestiziden). Im nationalen Aktionsplan Pflanzenschutz (NAP) sind bezüglich der Umsetzung des integrierten Pflanzenschutzes Zielquoten von 50\% Einhaltung der Leitlinien des integrierten Pflanzenschutzes nach fünf Jahren, bezogen auf ihr Veröffentlichungsdatum, genannt (Bundesministerium für Ernährung und Landwirtschaft 2017). Diese beziehen sich auf die allgemeinen Grundsätze. Diese acht Grundsätze lauten (gekürzt) wie folgt:

1. Einhaltung vorbeugender Maßnahmen (z. B. Fruchtfolge, Bodenbearbeitung)

2. Einsatz nicht-chemischer Methoden
3. z.B. Einsatz zielartspezifischer/nützlingsschonender Pflanzenschutzmittel

4. Verringerung der Aufwandmengen (z.B. Teilflächen), wenn möglich

5. Nutzung v. Resistenzvermeidungsstrategien

6. z.B. Überwachung des Anwendungserfolgs

7. Einsatz bzw. Bezug von Warndiensten u. Prognosemodellen

8. Anwendung von Schadschwellen

Zusätzlich wurden allgemeine Hemmnisse des IPS betrachtet.

Die wahrgenommene Umsetzung dieser Grundsätze soll für eine Stichprobe von 300 landwirtschaftlichen Betrieben aus Nordrhein-Westfalen, Schleswig-Holstein und Niedersachsen durch eine Befragung zum integrierten Pflanzenschutz erhoben werden.

\section{Material und Methoden}

\section{Fragebogen}

An der Fachhochschule Südwestfalen wurde am Fachbereich Agrarwirtschaft ein Fragebogen konzipiert, in welchem im Wesentlichen die acht Kernpunkte des IPS thematisiert wurden (BMEL 2020). Der Fragebogen wurde für eine computergestützte telefonische Befragung (computer assisted telephone interviews, CATI) entwickelt und durch das Marktforschungsinstitut Kleffmann in Lüdinghausen zunächst an 35 Personen getestet. Der Fragebogen gliedert sich dabei im Wesentlichen in fünf Hauptbereiche, die wiederum themenspezifisch untergliedert sind. Die Hauptbereiche sind dabei:

1. Betriebsstrukturen: Anbauumfang, Produktionsausrichtung, Wirtschaftsweise

2. Pflanzenbau: Produktionsmethoden (Bodenbearbeitung, etc.), Kulturenspektrum

3. Pflanzenschutz: IPS, Bestandskontrollen, Probleme, biologische $u$. mechanische Methoden

4. Warndienste/Prognosemodelle: Apps, Beratungsbezug

5. Demografie: Alter, Geschlecht, Ausbildungsstand

Der Fragebogen enthielt 40 offene und 18 Fragen mit vorgegebenen Antwortkategorien. Der hohe Anteil an offenen Fragen mit möglichst neutralen Formulierungen wurde gewählt, um soziale Erwünschtheit beim Antwortverhalten zu reduzieren. Gänzlich kann diese Fehlerquelle jedoch nicht ausgeschlossen werden (Diekmann 2014). Die Interviews dauerten alle ähnlich etwa 25 min lang. 23 Fragen, welche sich konkret mit Maßnahmen des integrierten Pflanzenschutzes beschäftigen, wurden für die vorliegende Auswertung herangezogen, ergänzt um sechs Fragen zur 
Demografie. Die Daten der Befragten wurden nicht an die auswertende Arbeitsgruppe weitergeleitet. Daher erfolgte eine anonymisierte Verwendung der Daten.

\section{Befragungsregion}

Als Befragungsregion wurden in Absprache mit dem Projektförderer die Bundesländer Nordrhein-Westfalen (NRW), Niedersachsen (NDS) und Schleswig-Holstein (SH) herangezogen. Einerseits, da alle drei Bundesländer Offizialberatung durch die Landwirtschaftskammer erhalten, andererseits, da sich die Betriebe innerhalb dieser Bundesländer strukturell am ehesten ähneln, und so eine Befragung nicht nur auf ein Bundesland beschränkt bleibt.

\section{Stichprobe}

Die Stichprobe für die Befragung wurde als Quotenstichprobe konzipiert. Dabei erfolgte die Quotierung nach den folgenden Vorgaben: Berücksichtigung des Landesdurchschnitts (Betriebsgröße und Ausrichtung), konventioneller Ackerbau sowie keine Schwerpunktausrichtung auf Sonder- oder Dauerkulturen, um die wahrgenommene Umsetzung des IPS in der Fläche erfassen zu können. Die Quotierung erfolgte, um zum einen eine gewisse Repräsentativität der Daten zu gewährleisten (Größe und Schwerpunkt), aber auch, um die Methoden des IPS zu erfassen. Diese gelten für den konventionellen Anbau, da der ökologische per se als integriert gilt. Auch die Betrachtung von Sonderkulturen könnte hier eine Verzerrung herbeiführen, da hier spezialisierte Methoden auf einer verhältnismäßig kleinen Fläche genutzt, für die Flächenkulturen mit hohem Anbauanteil jedoch keine Aussagen getroffen werden können. Der Stichprobenumfang umfasste 300 konventionelle landwirtschaftliche Betriebe.

\section{Durchführung}

Die Befragung wurde über vier Wochen von Anfang September bis Anfang Oktober 2019 durchgeführt und umfasste 300 landwirtschaftliche Betriebe in der Befragungsregion. Die Durchführung der CATIs erfolgte durch das Marktforschungsunternehmen Kleffmann in Lüdinghausen. Einige Fragen ermöglichten Mehrfachantworten. Diese wurden in ihrer Reihenfolge gelistet erfasst.

\section{Auswertung}

Die Auswertung der durch Kleffmann erhobenen Rohdaten erfolgte durch die Fachhochschule Südwestfalen. Zur Auswertung wurden die Programme Microsoft Excel 2016 und IBM SPSS Statistics Version 21 genutzt. Aufgrund der Quotenstichprobe als nicht-probabilistisches Verfahren zur
Stichprobenbildung erfolgte lediglich eine deskriptive statistische Auswertung ohne Verwendung von inferenzstatistischen Methoden. Dabei wurden teilweise Mittelwerte und Standardabweichung, in jedem Fall aber relative Häufigkeitsverteilungen zu den jeweiligen Fragen berechnet. Unter Berücksichtigung des Stichprobenverfahrens können damit lediglich begrenzt Rückschlüsse auf die angestrebte Grundgesamtheit aller Betriebe in den drei betreffenden Bundesländern gezogen werden. Mit den verwendeten Programmen wurden ebenfalls die Grafiken erstellt. Die als offen formulierten Fragen wurden während der Interviews direkt inhaltlichen Kategorien zugeordnet sowie die Nennungsreihenfolge dokumentiert, um sie statistisch auswerten zu können. Dazu kam es besonders auf die Fähigkeiten der InterviewerInnen an. Die MitarbeiterInnen der Fa. Kleffmann werden regelmäßig zu landwirtschaftlichen Themen geschult. Eine spezielle Einweisung auf die jeweilige Umfrage erfolgte für die beteiligten MitarbeiterInnen nach direktem Austausch mit der FH Südwestfalen. Fragen aus dieser Einweisung wurden an die FH zurückgegeben und beantwortet.

Die in der Durchführung erfassten Mehrfachantworten wurden in der Auswertung parallel betrachtet, d.h. ihre Verteilung gestapelt dargestellt. Es ist davon auszugehen, dass erste Antworten die durch die Befragten wahrgenommene höchste Relevanz bedeuten, die darauffolgenden Zweitund Drittantworten abnehmende wahrgenommene Relevanz besitzen. Diese Annahme entstand analog zur top-of-mind Befragung zu Marktpräferenzen (vgl. Esch 2021). Es kann sich jedoch auch um schlichte Aufzählungen handeln. Erstund nachfolgende Antworten sind daher gestapelt, um eine Gesamtzahl und eine differenzierte Betrachtung zu ermöglichen. Die Zweitantworten wurden aufgeführt, wenn innerhalb der Frage oder der befragten Gruppe wenigstens $20 \%$ der Stichprobe eine zweite Antwort abgegeben haben. Angegebene prozentuale Ergebnisse beziehen sich immer auf die ausgewiesene Zahl $n$. Ist diese nicht gesondert angegeben, gilt die Anzahl aller Befragten von $n=300$.

\section{Ergebnisse}

Die Ergebnisse werden in der bereits in der Einleitung angeführten Reihenfolge der acht Grundsätze vorgestellt. $\mathrm{Zu}$ jedem Grundsatz werden Fragen aus dem Fragebogen aufgenommen, welche Hinweise auf die Anwendung des Grundsatzes liefern können. Eine umfassende Vollständigkeit aller Aspekte, insbesondere verschiedener Nebenaspekte der Grundsätze, ist daher nicht gegeben, es können nur Tendenzen angegeben werden.

Grundlegende betriebsstrukturelle Kennzeichen und Merkmale der Betriebsleitenden sind in Tab. 1 ersichtlich. 
Tab. 1 Charakterisierung der Stichprobe im Vergleich zum gewichteten Durchschnitt der Bundesländer Nordrhein-Westfalen (NRW), SchleswigHolstein (SH) und Niedersachsen (NS) anhand der relativen Häufigkeiten bzw. dem Mittelwert mit Standardabweichung ( \pm ). (Quellen (verändert nach): LWK NRW 2017, Statistisches Amt für Hamburg und Schleswig-Holstein 2017+2019, Niedersächsisches Ministerium für Ernährung, Landwirtschaft und Verbraucherschutz (2020), Statistisches Bundesamt (2017))

\begin{tabular}{llll}
\hline & & Stichprobe & Gewichteter Landesschnitt (NRW, SH, NDS) \\
\hline Geschlecht & Weiblich & $2 \%$ & $8 \%$ \\
Alter (Jahre) & Männlich & $98 \%$ & $92 \%$ \\
& $>45$ Jahre & $18 \%$ & $22 \%$ \\
& $45-55$ Jahre & $29 \%$ & $38 \%$ \\
& $<55$ Jahre & $53 \%$ & $40 \%$ \\
Betriebsgröße (ha) & Durchschnitt (Jahre) & $53( \pm 12,5)$ & - \\
Schwerpunkt & - & $108,8( \pm 140)$ & 64,8 \\
& Marktfrucht & $46 \%$ & $32 \%$ \\
& Veredelung & $20 \%$ & $12 \%$ \\
Herkunft & Futterbau & $14 \%$ & $42 \%$ \\
& Gemischt & $20 \%$ & $14 \%$ \\
NDS & $46 \%$ & - \\
Angebaute Kulturen & NRW & $41 \%$ & - \\
Ausbildungstand & SH & $13 \%$ & - \\
& - & 3,8 & $53 \%$ \\
& Ausbildung & $10 \%$ & $22 \%$ \\
& Meister & $26 \%$ & $16 \%$ \\
& Techniker & $37 \%$ & $9 \%$ \\
\hline
\end{tabular}

\section{Einhaltung vorbeugender Maßnahmen}

Zunächst wird der Grundsatz der Vorbeugung von Schadorganismen anhand zweier Fragenkomplexe, nämlich „Worauf achten Sie bei der Auswahl Ihrer ... Sorten?“, sowie „Welche Bodenbearbeitungsform setzen Sie hauptsächlich auf Ihrem Betrieb ein?" näher betrachtet. Dabei wird die Sortenwahl als vorbeugendes Kriterium für Pilzerkrankungen betrachtet, die Bodenbearbeitung sowohl zur vorbeu- genden Bekämpfung pilzlichen Inokulums als auch gegen Unkräuter und Gräser.

Bezüglich der Sortenwahl wurden die Teilnehmer nach Getreide, Mais, Raps, Kartoffeln und Rüben befragt, sofern diese im jeweiligen Anbauspektrum vorkamen. Daraus resultierten die folgenden Ergebnisse (Abb. 1):

Die Gesundheit der Sorte, ob gegen pilzliche Erreger, Virusanfälligkeiten o. ä. findet besonders bei Getreide und Rüben nach Angabe der Betriebsleiter Beachtung in der Sortenwahl. Bei Kartoffeln sind vor allem Qualität und
Abb. 1 Sortenwahlkriterien von Getreide, Raps, Rüben, Mais und Kartoffeln in ihrer prozentualen Verteilung

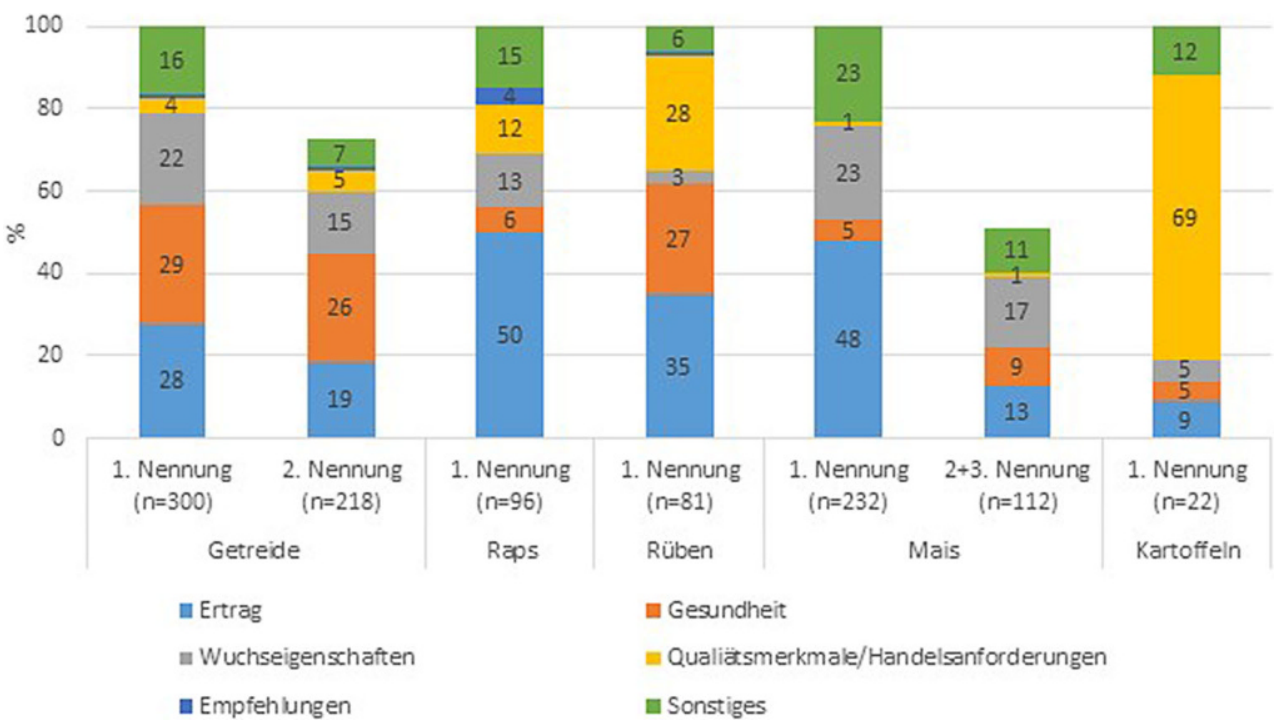


Abb. 2 Angaben zu den auf den Betrieben überwiegend eingesetzten Formen der Bodenbearbeitung

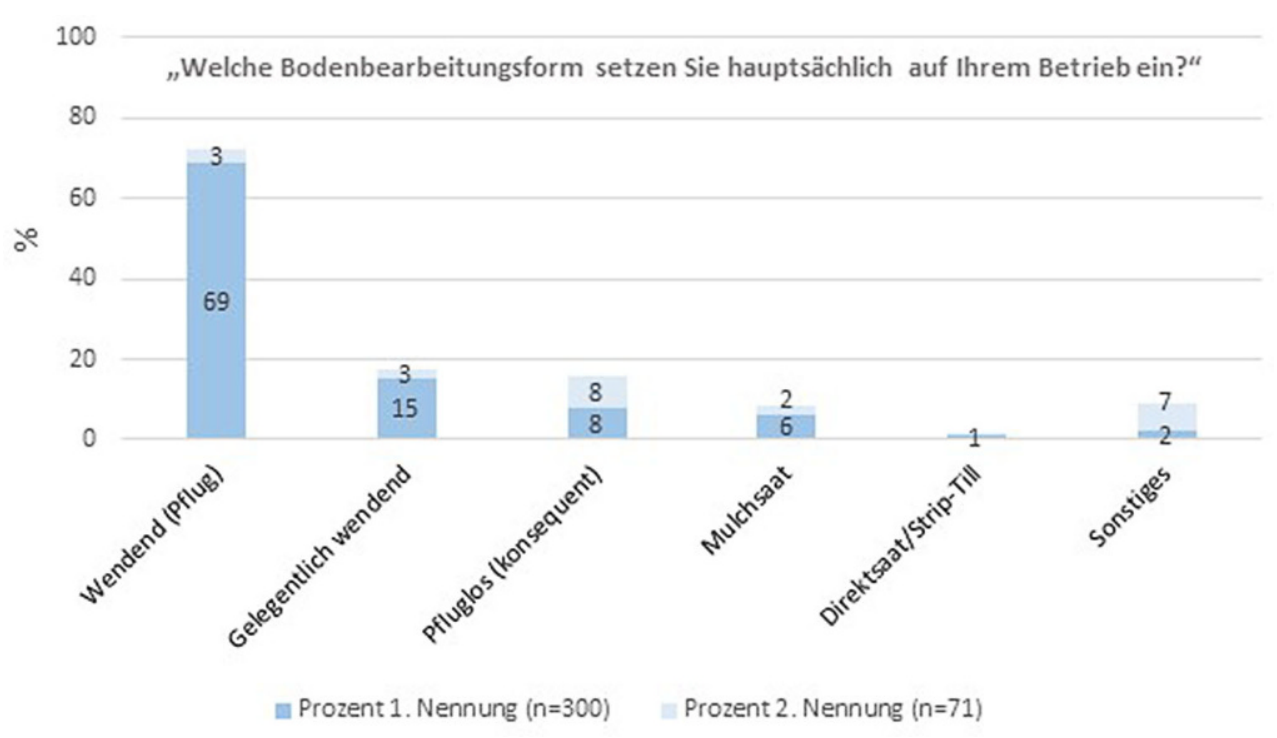

Handelsanforderungen maßgeblich für die Auswahl der angebauten Sorte. Der Ertrag ist für die Wahl von Mais- und Rapssorten entscheidender als andere Faktoren und ebenfalls entscheidender als bei anderen Kulturen. Unter den Wuchseigenschaften im Getreide wurde häufig das Längenwachstum genannt. Hier können sekundäre Einflussfaktoren auf Krankheitsanfälligkeiten mit angenommen werden. $\mathrm{Zu}$ beachten ist, dass für Mais und Getreide eine nicht unerhebliche Anzahl an Zweit- und Drittnennungen zusammengekommen ist. Aufgrund der insgesamt geringen Mehrfachantworten ist eine differenzierte Betrachtung von Erstund Zweit/Drittantworten ggf. sinnvoll, da es sich nicht um große Aufzählungen von Eigenschaften handelt, sondern durch die Befragten nur eine kleine Anzahl genannt wurde. Es ist daher nicht auszuschließen, dass die genannten Eigenschaften in ihrer Aufzählungsreihenfolge unterschiedlich stark für die Auswahl der Sorte gewichtet werden.

Die Bodenbearbeitung erfolgte gemäß Angaben schwerpunktmäßig wendend, also durch den Pflug (Abb. 2).

Aus Abb. 2 geht hervor, dass summiert (1. Nennung) auf etwa über $70 \%$ der Betriebe der Pflugeinsatz als regelmäßig angegeben wird. Pfluglose Systeme sind dagegen in der 1. Nennung mit $15 \%$ vertreten.

\section{Einsatz nicht-chemischer Methoden}

Der Einsatz nicht-chemischer Methoden im Pflanzenschutz bezieht sich auf die Bereiche des Insektizid-, Fungizidund Herbizideinsatzes. Exemplarisch werden hier die Alternativmethoden zum chemischen Herbizideinsatz betrachtet, da hier Techniken für alle betrachteten Kulturen/ Kulturengruppen vorhanden sind. Die relative Häufigkeit, mit welcher in den Kulturen mechanisch Unkräuter bekämpft werden, ist in Abb. 3 dargestellt.
Der überwiegende Teil der Befragten gibt keine gezielten Maßnahmen gegen Unkräuter und Gräser in den angebauten Kulturen an. Es zeigt sich, dass Getreide als Kulturengruppe, welche auf jedem Betrieb angebaut wird, von $12 \%$ der Betriebe regelmäßig durch mechanische Methoden gepflegt wird. Bezogen auf den Umfang der befragten Anbauer setzten nach eigenen Angaben 12,5\% der Maisanbauer und $26 \%$ der Rübenanbauer regelmäßig mechanische Methoden zur Unkrautregulierung ein, ebenso $12 \%$ der Kartoffelanbauer. Im Raps als urtümliche Hackfrucht wird hingegen von $2 \%$ der Rapsanbauern Hacke oder Striegel eingesetzt.

Nicht nur die aktuelle Situation kann mögliche Hinweise auf die Umsetzung des IPS bezogen auf nicht-chemische Maßnahmen liefern, sondern auch die Bereitschaft, zukünftig intensiver den Einsatz mechanischer Methoden auf den Betrieben einzusetzen. Dazu erfolgte eine Frage zur Planung bezüglich einer Anschaffung von Hacke oder Striegel auf den teilnehmenden Betrieben. In Abb. 4 sind die Ergebnisse dargestellt. Insgesamt zeigt sich eine zu $74 \%$ ablehnende und eine zu $13 \%$ zustimmende Haltung.

Biologische Methoden zur Schädlingsabwehr wurden überwiegend in Mais und Getreide angegeben, sofern sie zum Einsatz kamen. $86 \%$ der befragten Betriebe geben an, keine biologischen Methoden in den vergangenen drei Jahren eingesetzt zu haben (Abb. 5.) Im Mais wurde neben dem Einsatz mechanischer Beikrautregulierung Trichogramma gegen Maiszünsler angegeben. Die Antworten in den übrigen Kulturen entfielen auf mechanische Regulierung durch Striegel und Hacke.

\section{Einsatz zielartenspezifischer/nützlingsschonender Pflanzenschutzmittel}

Die Befragung und Erfassung innerhalb der Stichprobe zum Einsatz nützlingsschonender/zielartenspezifischer Pflanzen- 
Abb. 3 Angaben zum Einsatz regelmäßiger mechanischer Unkrautbekämpfung in verschiedenen Kulturen
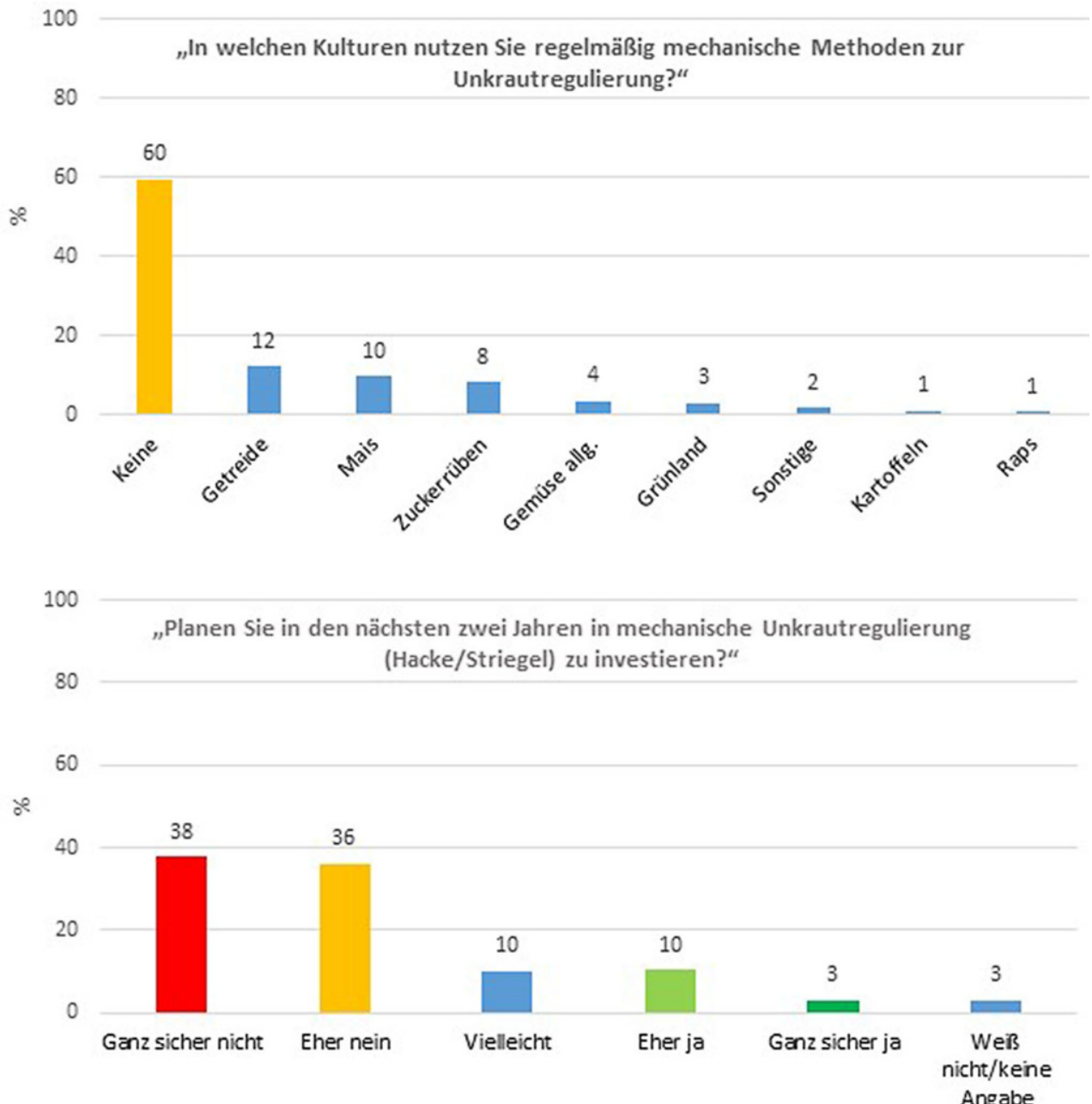

Abb. 4 Angaben zur Bereitschaft der befragten Betriebe, innerhalb der nächsten zwei Jahre in mechanische Unkrautbekämpfung (Hacke/Striegel) zu investieren

Abb. 5 Angaben zum Nutzen biologischer Methoden zur Schädlingsabwehr innerhalb der letzten drei Jahre auf den befragten Betrieben

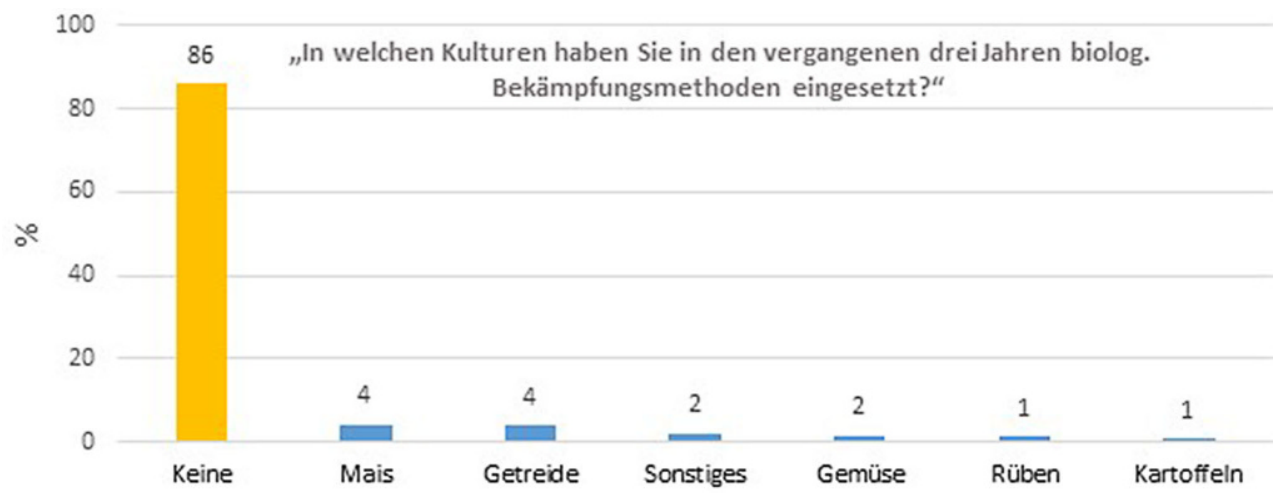

schutzmittel stellte die Urheber der Befragung vor Probleme, da sie nicht wörtlich abgefragt wurde. So erfolgte auch für diese Frage eine Näherung über den Einfluss auf die Präparatewahl (Abb. 6).

Das Charakteristikum der Umweltverträglichkeit als Kriterium der Präparatewahl wirkt sich nach eigenen Angaben bei $35 \%$ der Betriebe sehr stark, bei $30 \%$ stark auf die Auswahl des zu verwendenden Produkts aus (Abb. 6).
Durch die Urheber wurden in diesem Fall „Umweltverträglichkeit" als Indiz für die Beachtung des Nützlingsschutzes herangezogen, da dieser Aspekt durch den Anwender am Produkt festgestellt werden kann. Gleichwohl kann die Umweltverträglichkeit weitreichendere Aspekte umfassen. Darüber hinaus zeigt die Abb. 6 einen starken Einfluss der Wirksamkeit $(60 \%)$ sowie der produktbezogenen Anwendungsauflagen $(50 \%)$ auf die Auswahl. Eine weitere wich- 
Abb. 6 Angaben zur Einflussintensität verschiedener Faktoren auf die Auswahl von Pflanzenschutzprodukten arozent 1. Nennung $(n=300) \quad$ Prozent 2. Nennung $(n=61)$ Pflanzenschutzmittel?"
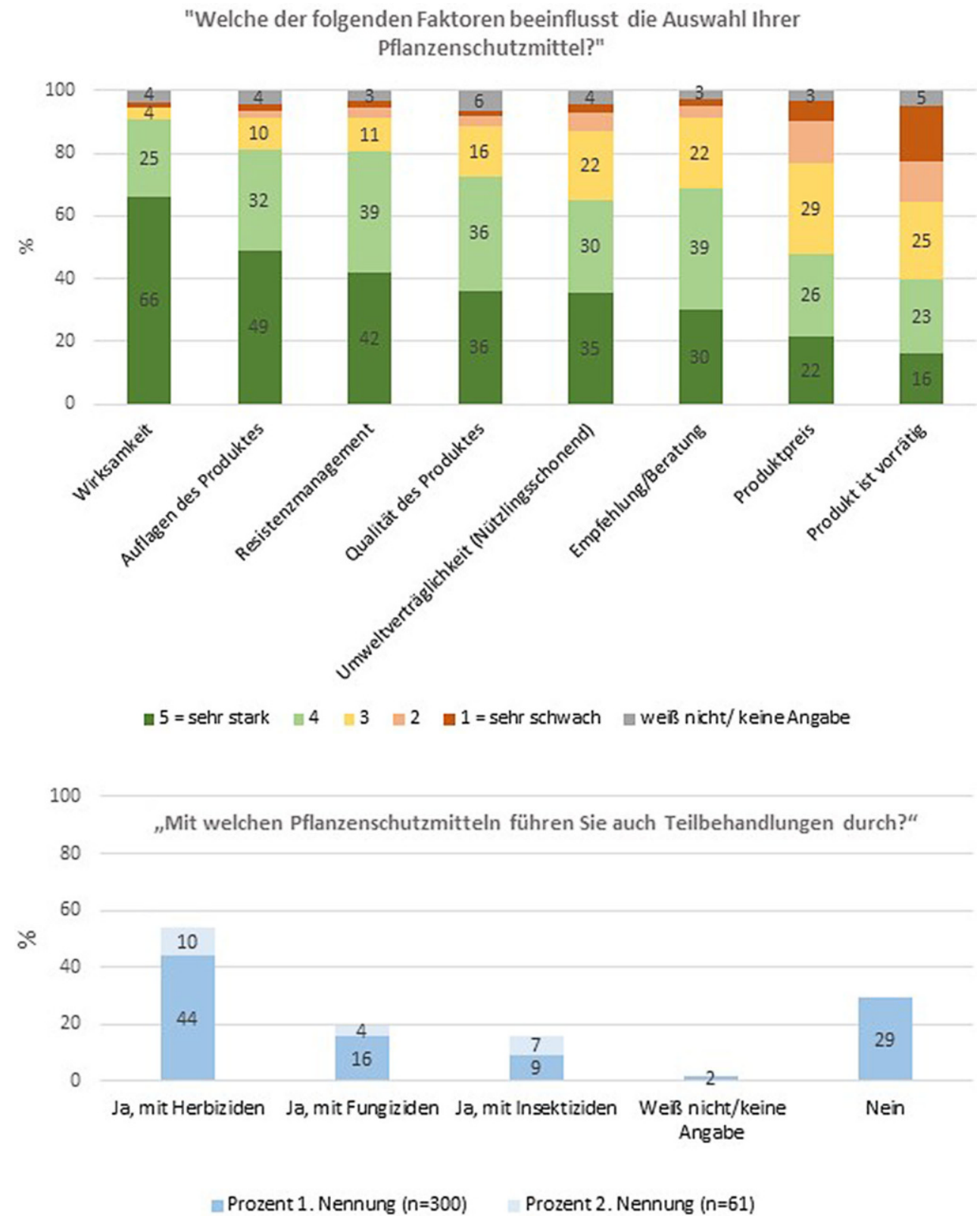

Abb. 7 Angaben zur Durchführung von Teilbehandlungen mit Herbiziden, Fungiziden und Insektiziden auf den befragten Betrieben
"Welche der folgenden Faktoren beeinflusst die Auswahl Ihrer

tige Rolle wird den Angaben zu Folge dem Resistenzmanagement (42\%) eingeräumt. Deutlich weniger Einfluss scheinen die Faktoren der Vorrätigkeit und des Produktpreises zu spielen. Die Beachtung von Beratung und Nützlingsschutz/ Umweltschutz liegen im mittleren Bereich (Abb. 6).

\section{Verminderung der Aufwandmenge (Teilflächenapplikation)}

Die Reduktion der ausgebrachten Menge kann einerseits über die Verminderung der Aufwandmenge des Pflanzenschutzmittels erfolgen, oder über die spezifische Applikation auf Teilflächen, auf welchen der Schaderreger auftritt. Diesen Aspekt beleuchtet Abb. 7.
Knapp ein Drittel der teilnehmenden Betriebe führt nach eigenen Angaben keine Teilflächenbehandlung mit Pflanzenschutzmitteln durch. Teilapplikationen mit Herbiziden werden von $54 \%$ der Befragten durchgeführt, $20 \%$ gaben an, auch mit Fungiziden Teilflächenapplikationen durchzuführen. Insektizide werden auf etwa $87 \%$ der Betriebe bei einem Einsatz flächig genutzt. Zwei Prozent der Betriebe machten zu dieser Frage keine Angaben.

\section{Nutzung von Resistenzvermeidungsstrategien}

Die Anwendung von Resistenzstrategien wurde nicht gezielt durch die Befragung erfasst, wahrgenommene Probleme mit Resistenzen hingegen schon. Das Bewusstsein über 
Abb. 8 Anteil der Befragten mit wahrgenommenen Bekämpfungsproblemen von Schadinsekten in verschiedenen Kulturen
Abb. 9 Angaben zu wahrgenommenen Problemen bei der Bekämpfung von Pilzerkrankungen in verschiedenen Kulturen
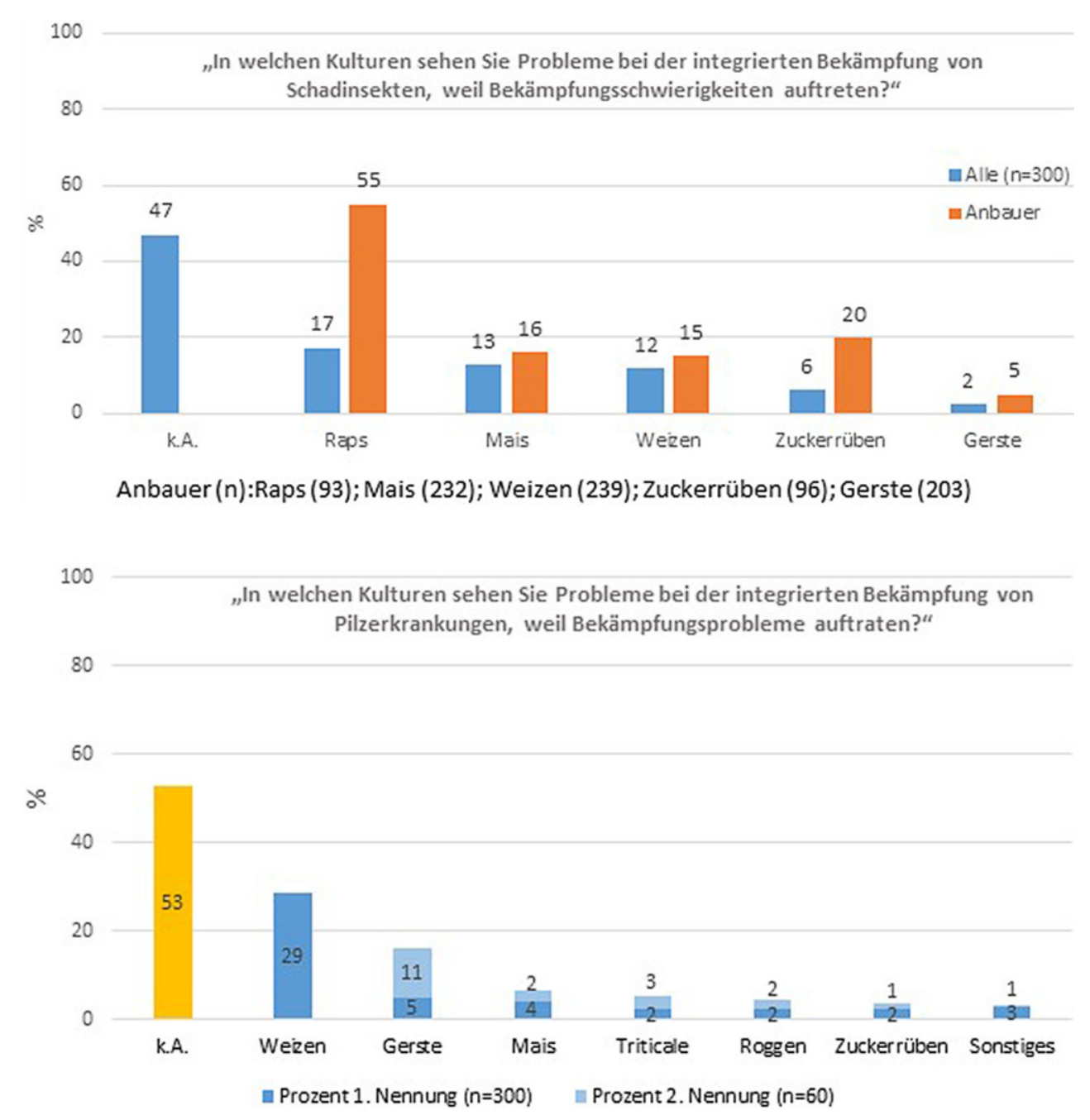

Bekämpfungsschwierigkeiten auf dem eigenen Betrieb und den eigenen Flächen lässt u. U. die Sensibilität für die Notwendigkeit einer Resistenzstrategie steigen. Erfasst wurden wahrgenommene Bekämpfungsprobleme für die Bereiche der Herbizide, der Insektizide und der Fungizide zum Einsatz auf der Ackerfläche. Dabei wurde durch den Fragebogen nur das wahrgenommene Problem, nicht aber der problematische Schaderreger erfasst. Gleichzeitig wurden keine Gegenmaßnahmen, welche durch die Betriebe möglicherweise ergriffen wurden, erfasst. Der Einfluss des Resistenzmanagements auf die Entscheidung der Präparatewahl hingegen ist erfasst und in Abb. 7 aufgeführt.

Abb. 8 zeigt, dass eine große Gruppe innerhalb aller Befragten keine Bekämpfungsprobleme bezüglich Schadinsekten wahrgenommen oder eine intensivere Auseinandersetzung mit der Thematik vollzogen hat. Etwa $17 \%$ sehen den Raps und damit verbunden die Rapsschädlinge und etwa $13 \%$ die Maisschädlinge als problematisch in ihrer Bekämpfung an.

Probleme bei der Bekämpfung pilzlicher Erreger werden hingegen überwiegend im Weizen durch die Befrag- ten wahrgenommen. Mit Abstand folgt die Gerste. Wie auch schon in Abb. 6 zeigt sich, dass etwa die Hälfte der Befragten keine Bekämpfungsschwierigkeiten wahrgenommen bzw. kommuniziert hat (Abb. 9).

Für Herbizide und wahrgenommene Bekämpfungsprobleme von Unkräutern und Gräsern zeichnet sich ein anderes Bild ab. Hier geben nur 22\% der Befragten an, keine Probleme wahrzunehmen. Aufsummiert kommen $31 \%$ der Befragten zu der Aussage, dass sie Bekämpfungsschwierigkeiten bzw. wahrgenommene Resistenzen auf ihren Flächen vorfinden.

\section{Überwachung des Anwendungserfolgs}

Die Erfolgskontrollen werden in den acht allgemeinen Grundsätzen des IPS an sechster Stelle aufgeführt. Sie erfolgen nach dem Einsatz chemischer und nicht-chemischer Maßnahmen in den Kulturen. Dabei werden Unterschiede bezüglich Art und Verteilung der Erfolgskontrollen zwischen den Gruppen der Fungizide, Herbizide und Insektizide deutlich, wie aus Abb. 10 hervorgeht. 
Abb. 10 Angaben zu Erfolgskontrollen von Pflanzenschutz auf die Gruppen Herbizide, Insektizide und Fungizide bezogen auf die behandelte Fläche
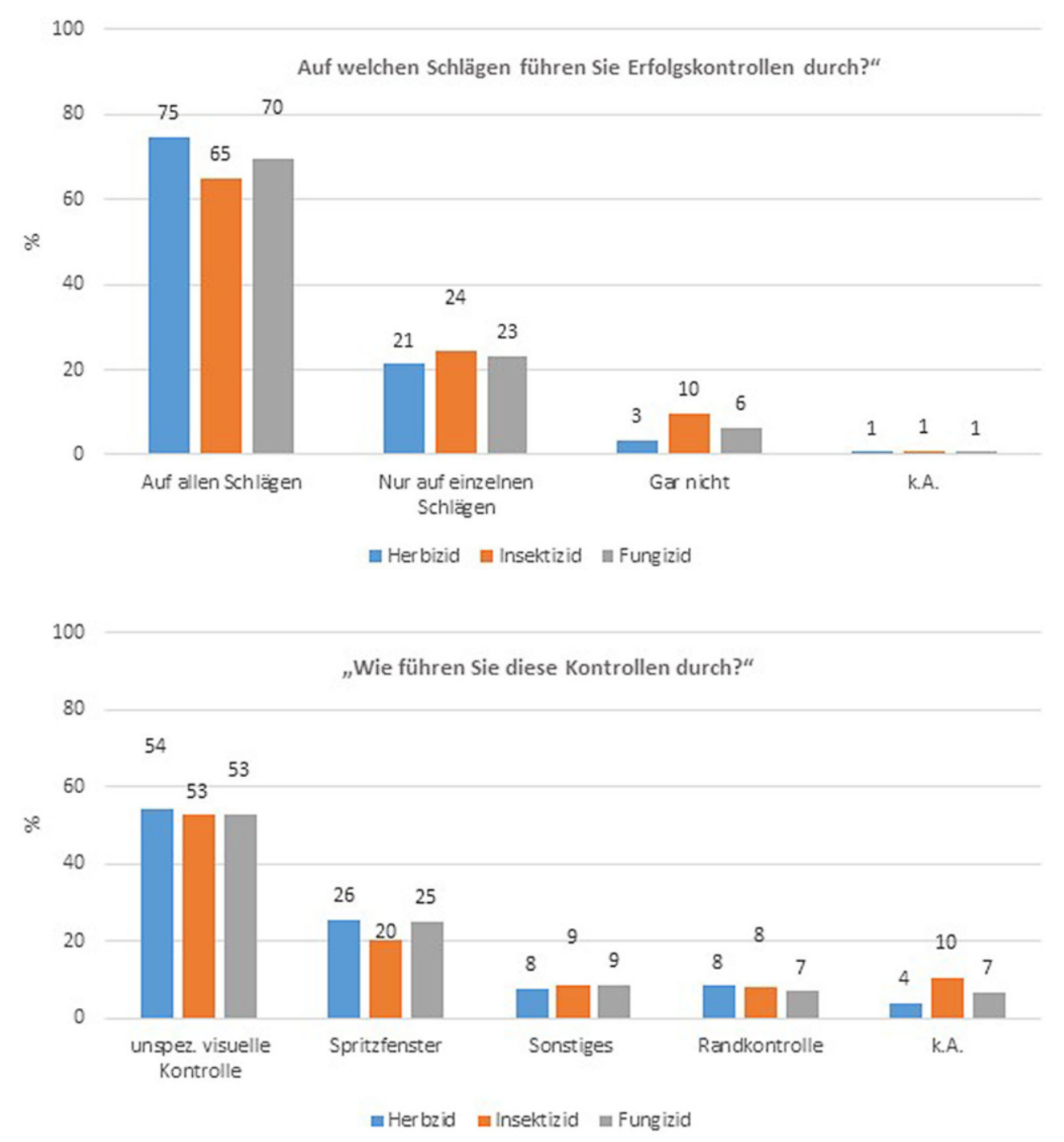

Abb. 11 Angaben zur Art der Feldkontrolle nach erfolgter Applikation von Herbiziden, Insektiziden und Fungiziden
Abb. 11 zeigt, dass besonders nach Herbizideinsatz eine Erfolgskontrolle stattfindet. Dies wird ebenso deutlich am geringen Anteil von 3,3\% der Betriebe, welche nach eigenen Angaben keine Erfolgskontrollen bezogen auf den Herbizideinsatz durchführen. Dieser Wert ist mit $10 \%$ am höchsten für die Insektizide.

Wie schon zuvor in der Verteilung der Erfolgskontrollen auf die drei Gruppen Herbizid, Insektizid und Fungizid, zeigt sich auch in der Art der Kontrolle eine negative Tendenz für die Kontrolle von Insektizideinsätzen. Zunächst geben über $50 \%$ der Befragten an, unspezifische visuelle Kontrolle innerhalb des Schlags, durch die Befragten selbst häufig als „mal drüber gucken“ bezeichnet, durchzuführen. Randkontrollen werden auf 7-8\% der Betriebe durchgeführt. Spritzfenster werden nach Angaben der Betriebe für Herbizide und Fungizide (beides $25 \%$ ) und Insektizide genutzt. Die Erfolge der Insektizide werden jedoch um 5\% weniger in Spritzfenstern kontrolliert.

\section{Einsatz bzw. Bezug von Warndiensten und Prognosemodellen}

Um Schadorganismen gezielt und effektiv auf der Fläche einzudämmen bzw. zu vermeiden, lässt sich neben digitalen Hilfsmitteln wie Prognosesystemen verschiedener Hersteller auch die Beratung hinzuziehen. Nur etwa $20 \%$ der teilnehmenden Betriebe konnten jedoch Anwendungserfahrungen mit Prognosemodellen vorweisen.

Als Bezug für qualifizierte Beratung wird vor allem auf die Landwirtschaftskammer als Offizialberatung der drei Bundesländer zurückgegriffen (Abb. 12). Der Anteil der Betriebe, welche überhaupt keine Beratung beziehen, und sich diese dem Antwortenspektrum nach auch nicht über andere Wege verschaffen, liegt bei gerundeten $14 \%$.

Der bezogenen Beratung wird ebenfalls ein Einfluss auf die Entscheidungen im Pflanzenschutz zugeschrieben. Einen etwa gleichgroßen Einfluss nehmen dabei Landhandel und Offizialberatung auf Entscheidungen ein, gefolgt 
Abb. 12 Art der bezogenen Beratung auf den befragten Betrieben
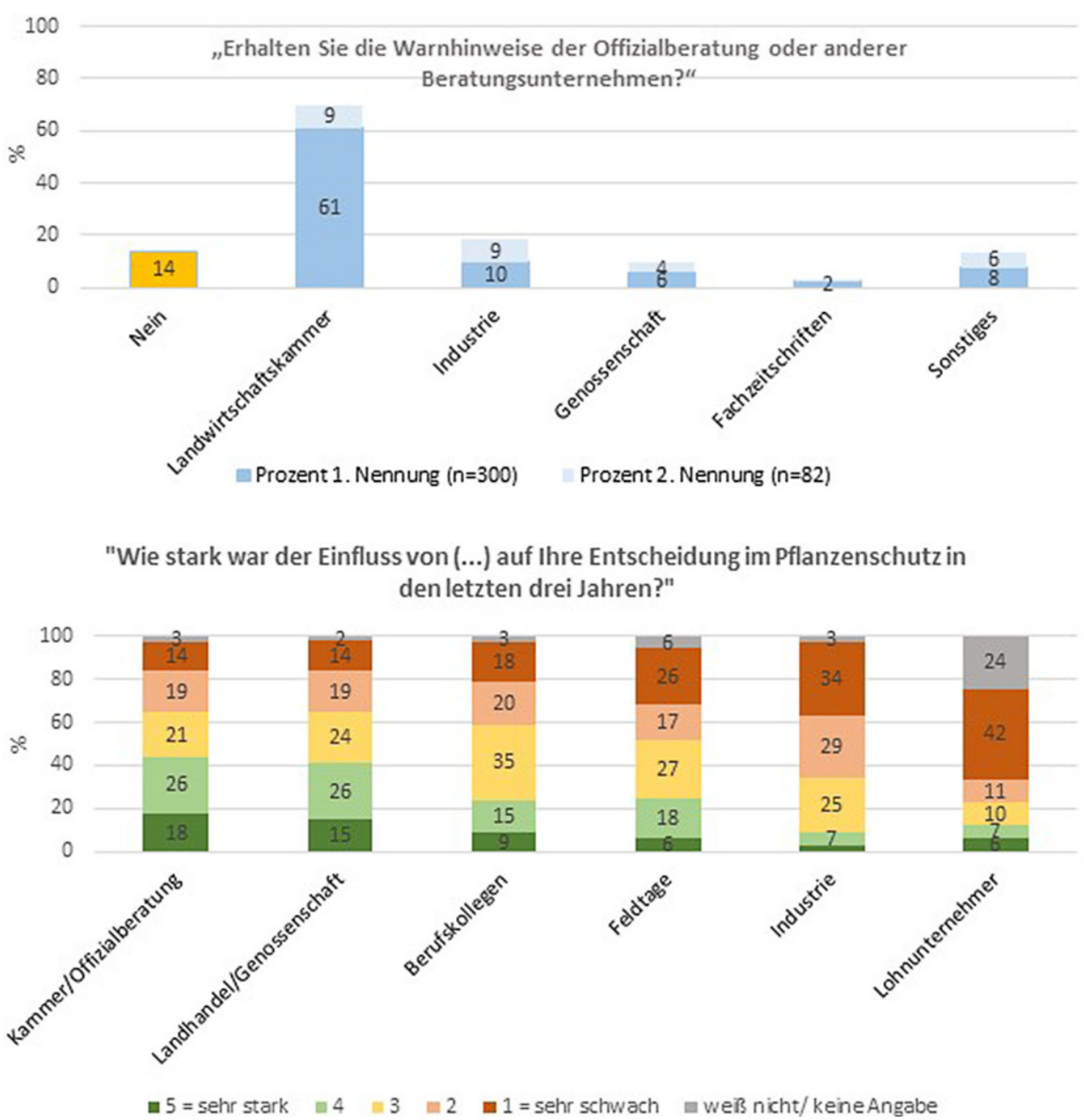

Abb. 13 Wahrgenommene Einflussintensität verschiedener Faktoren auf die Entscheidungsfindung bezogen auf Pflanzenschutzmittelanwendungen von Berufskollegen und Feldtagen. Lohnunternehmen oder Industrie haben nach Angaben der Befragten ein geringerer Einfluss auf die Entscheidungen auf den Betrieben (Abb. 13).

\section{Anwendung von (und Erfahrungen mit) Schadschwellen}

Letzter der acht allgemeinen Grundsätze des IPS ist die Anwendung von Schadschwellen zur Entscheidungsfindung, sofern für den jeweiligen Schaderreger der regionalspezifische Schadschwellenwert angegeben ist. Innerhalb der Befragung wurde besonders auf Probleme bzw. Kritik an Schadschwellen eingegangen. Diese wurden entweder erregerspezifisch (Schadinsekten) oder kulturspezifisch erfasst. Abb. 14 zeigt solche Insekten, deren Schadschwellen durch Landwirte überwiegend als kritisch betrachteten wurden.

Ein Drittel der Befragten geben keine Probleme mit den geltenden Schadschwellen an. Blattläuse und Maiszünsler wurden am häufigsten und zweithäufigsten genannt. Für pilzliche Erreger wurde nach Kulturen gefragt, in denen die befragten Betriebe wahrgenommene Probleme mit der Anwendung von Schadschwellen festgestellt haben. Dabei fällt auf, dass etwa die Hälfte in keiner angebauten Kultur die Schadschwelle als kritisch betrachtet. Im Getreide, besonders im Weizen (31\%), werden häufiger Probleme wahrgenommen als etwa in Hackfrüchten (Abb. 15).

Die Schadschwellen für Unkräuter und Ungräser werden insgesamt von $6 \%$ der befragten Betriebe als kritisch betrachtet. Eine differenziertere Befragung erfolgt in diesem Bereich nicht.

Hilfsmittel, um die verschiedenen Schadorganismen zu erfassen, werden auf den Betrieben unterschiedlich intensiv genutzt. Etwa $60 \%$ nutzen für die Feldkontrollen auf Unkräuter und Gräser keine Hilfsmittel, Zählrahmen und Prognosemodelle werden von $13 \%$ bzw. $11 \%$ der Befragten angegeben (Abb. 16).

Für die Kontrolle auf Schadpilze und Schadinsekten zeigt sich ein differenziertes Bild. So verwenden hier nur etwa $50 \%$ der Befragten keine Hilfsmittel, $31 \%$ gab das Nutzen von Gelbschalen an (Abb. 17). Bezogen auf alle befragten Rapsanbauer ergibt dies eine Nutzung von $67 \%$. 
Abb. 14 Schadinsekten, deren Schadschwellen als kritisch betrachtet werden
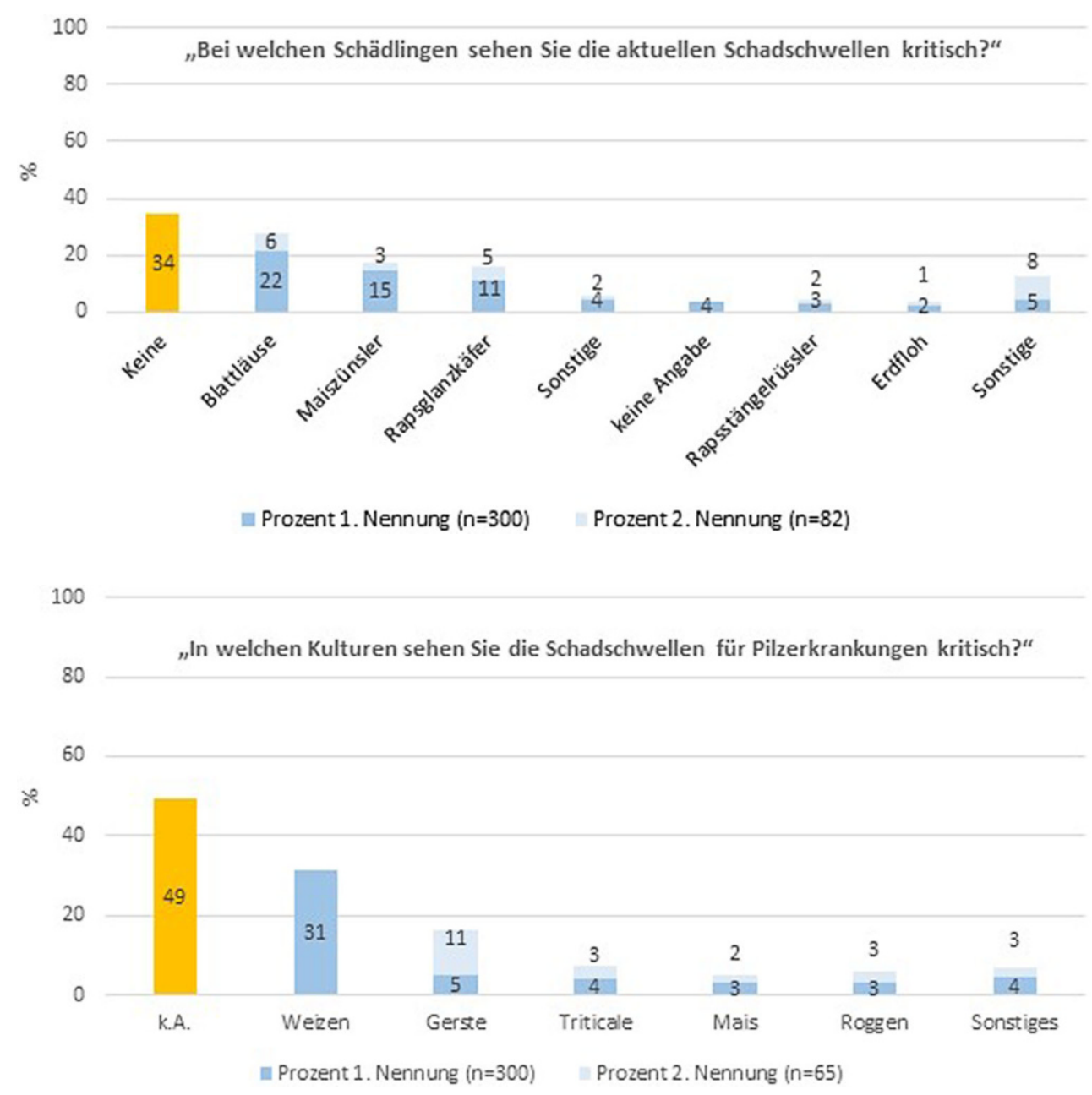

Abb. 15 Verteilung der Kulturen, in welchen die Schadschwelle für Pilzerkrankungen als kritisch wahrgenommen wird

Abb. 16 Hilfsmittel zur Erfassung des Unkrautaufkommens bei der Feldkontrolle

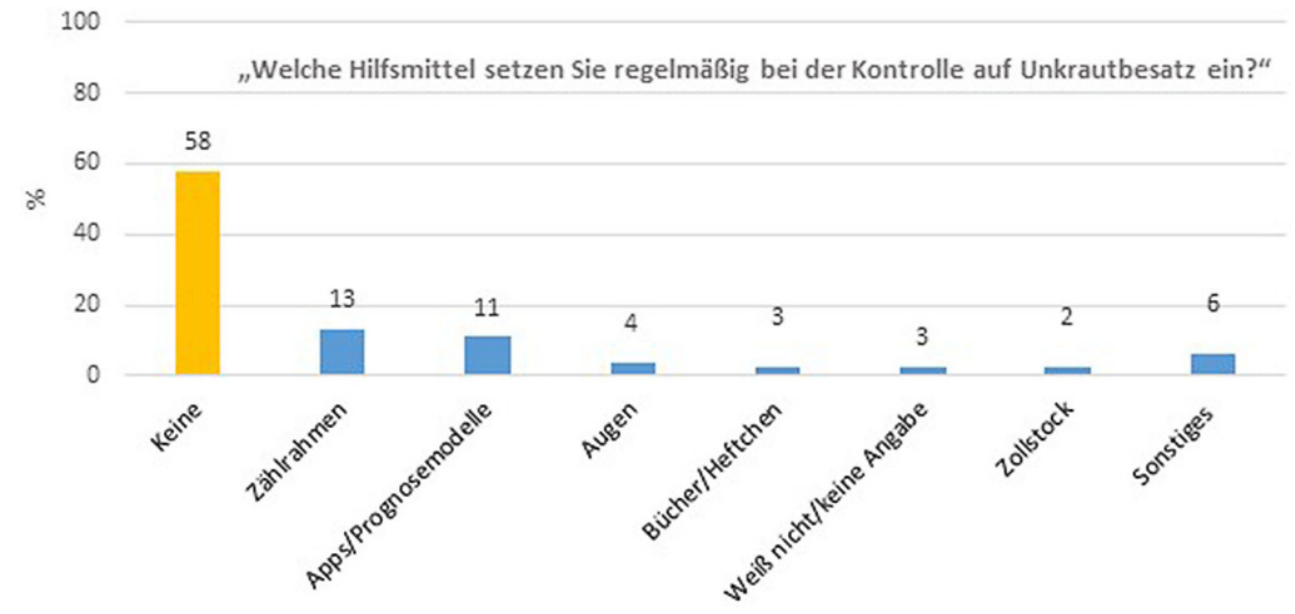

\section{Hemmnisse im IPS}

Betrachtet man abschließend die Hemmnisse, welche bei der Umsetzung des IPS insgesamt wahrgenommen werden, so fällt auf, dass insbesondere der Zeitaufwand und das wahrgenommene Risiko durch die verschiedenen Schaderreger von über $50 \%$ der befragten Betriebe als Hemmnisse betrachtet werden. Ebenfalls spielen Probleme bei der Erfassung (und dem Erkennen) sowie eine kritische Haltung gegenüber den bekannten Schadschwellen (17\%) eine deutliche Rolle (Abb. 18). 
Abb. 17 Nutzung von Hilfsmitteln zur Kontrolle von Schadpilzen und Schadinsekten

Abb. 18 Wahrgenommene Hemmnisse zur Anwendung des IPS

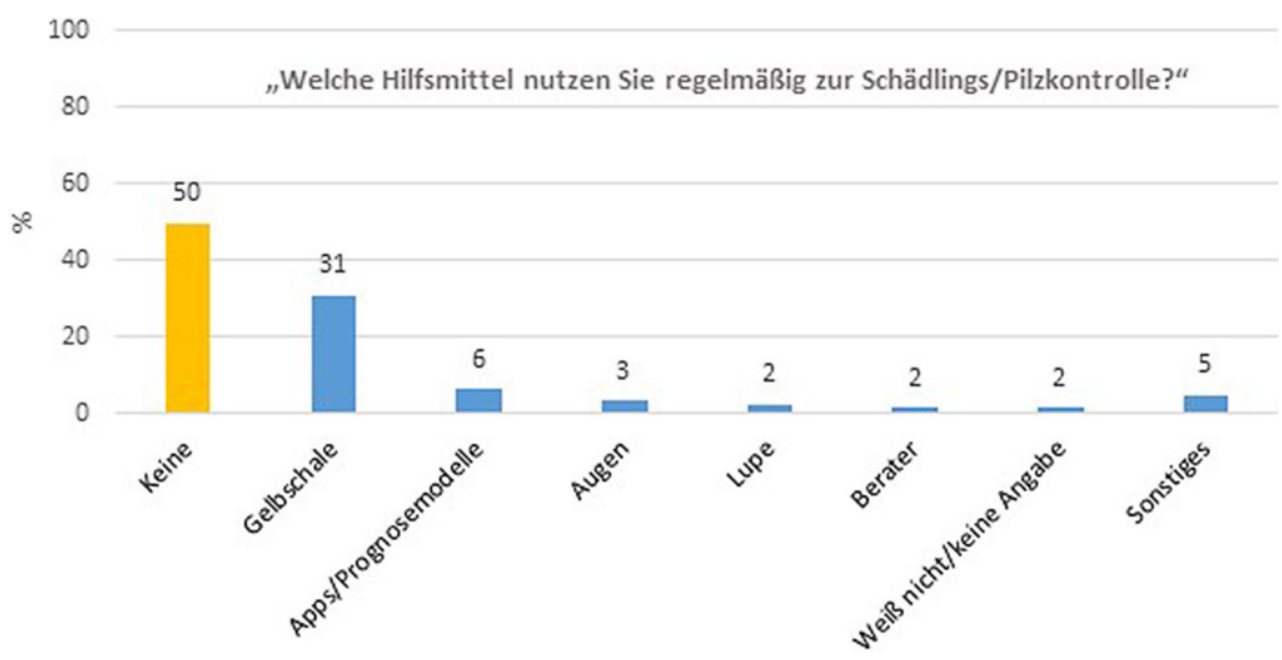

"Wo sehen Sie ganz allgemein Hemmnisse bei der Umsetzung des integrierten pflanzenschutzes?"

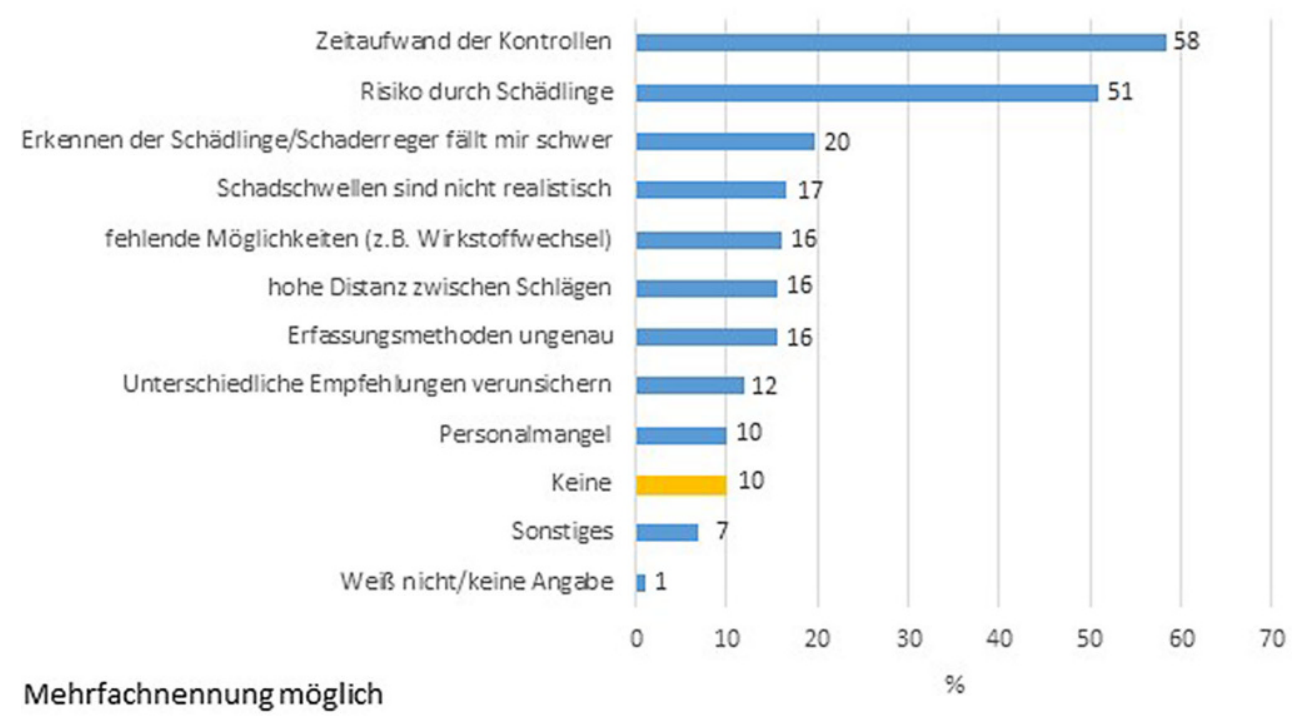

\section{Diskussion}

Die vorliegenden Daten zeigen Teilergebnisse einer umfangreichen Befragung von landwirtschaftlichen Betrieben aus Nordwestdeutschland. Die Daten können Teilaspekte bei der wahrgenommenen Umsetzung des IPS beleuchten.

1. Es zeigt sich, dass besonders in den Getreidekulturen eine Berücksichtigung des sortenspezifischen Gesundheitsstatus durch die Befragten gegeben ist. Auch bei Rüben wird der Gesundheitsstatus der Sorte gegenüber pilzlichen Erregern oder aber Nematoden beachtet. Für die übrigen Kulturen, etwa Raps, sind Krankheitsresistenzen scheinbar weniger entscheidend. Hintergrund ist möglicherweise, dass die beschreibende Sortenliste keine ausreichende Auskunft über Eigenschaften gegenüber diversen Krankheitserregern anbietet. (Bundes- sortenamt 2019a). Demgegenüber greifen Zuchtunternehmen Krankheitsresistenzen in der Kommunikation auf (z. B. Limagrain 2021). Krankheitsresistenzen können deshalb potenziell die Sortenentscheidungen auf Betrieben durchaus beeinflussen. Hilfreich könnte hier für Betriebsleitende eine stärker auf objektive Daten zurückgreifende Bewertung sein. Für die Anbauer von Kartoffeln liegen zwar Informationen zu Phytophtora infestans vor (Bundessortenamt 2019b), die Ergebnisse hier zeigen jedoch, dass Vermarktungsabsprachen und -vorgaben bei Kartoffeln einen deutlicheren Einfluss auf die Sortenwahl ausüben als bei anderen hier aufgeführten Kulturen. Durch die differenzierte Betrachtung von Erst- und Zweitnennungen bei Getreide und Mais zeigt sich, dass die Ertragseigenschaften besonders in der ersten Nennung höher sind und in den Zweitnennungen im Verhältnis zugunsten von Gesundheit und Wuchsei- 
genschaften abnehmen. Dies gibt Hinweis darauf, dass der wirtschaftliche Erfolg der Sorte weiterhin über den Ertrag definiert wird und weitere, für die Produktion vorteilhafte, Faktoren keine ausreichende Berücksichtigung finden. Es könnte jedoch auch sein, dass unter hohem Ertrag eine hohe Vitalität verstanden wird.

Isoliert und kurzfristig auf den integrierten Pflanzenschutz bezogen, ist der überwiegende Einsatz des Pflugs durchaus als positiv zu bewerten. Neben dem Verschütten von Unkräutern und deren Samen wird ebenfalls ein mögliches Inokulum an abgestorbenen Pflanzenresten aus dem Infektionshorizont für die Folgekultur entfernt. Langfristig ist die Gefahr für Bodenschadverdichtungen und damit eine folgende Schwächung des Pflanzenbestands erhöht (Estler 2011). Zudem werden in den acht allgemeinen Grundsätzen Systeme der Direktsaat und konservierenden Bodenbearbeitung bevorzugt. Insgesamt erscheinen vorbeugende Maßnahmen nach den Angaben in den Interviews bereits in Teilen umgesetzt zu werden.

2. Der Einsatz nicht-chemischer Methoden zur Regulierung von Schaderregern und Unkräutern zeigt auf den ersten Blick eine geringe Akzeptanz. Besonders der Einsatz von Trichogramma im Mais wurde innerhalb der Befragung als am verbreitetsten herausgestellt. Ein Grund für den übrigen, geringen Einsatz biologischer Verfahren ist wahrscheinlich das Fehlen vorhandener Möglichkeiten. Zwar liegen alternative Beizverfahren, etwa elektronische Beizung, vor (BayWa 2020), ein aktiver Einsatz von auch im biologischen Anbau zugelassenen Fungiziden ist bisher, neben den bekannten Varianten wie Kupfer oder Schwefel, kaum möglich, da nur wenige Präparate auf dem Markt verfügbar sind sowie den konventionellen Anbauer in ihrer Wirkung nicht überzeugen. Eine der wenigen Ausnahmen stellt hier Coniothyrium minitans dar (Zeng et al. 2012). Die geringe Bereitschaft zur Investition in mechanische Unkrautregulierung hängt möglicherweise mit hohen Anschaffungskosten für moderne, präzise Geräte zusammen und der noch begrenzten breiten praktischen Erfahrung mit diesen Geräten. Gleichzeitig sind betriebsindividuell die Möglichkeiten der Nutzung in Abhängigkeit von der Bodenart u. U. nur sehr begrenzt. In Anbetracht einer wirtschaftlich attraktiven und pflanzenbaulich sicheren Regulierungsmöglichkeit über Herbizide wirken diese Faktoren einzelbetrieblich hinderlich und lassen offen, ob und wie alternative Verfahren zur Unkrautregulierung ohne ordnungs- bzw. förderrechtliche Unterstützung stärker umgesetzt werden können.

3. Der Einsatz zielartenspezifischer Pflanzenschutzmittel wurde näherungsweise über das Auswahlkriterium „nützlingsschonend/umweltschonend“ betrachtet. Betrachtet man Fungizide und Herbizide, aber auch Insek- tizide, so wird deutlich, dass die zugelassenen Produkte stets eine Wirkung gegen mehrere Organismen erzielen. Eine völlige Beschränkung auf einen ausgewählten Zielorganismus ist dem Anwender folglich nicht möglich oder sinnvoll. Der Schutz von Nützlingen durch die Auswahl geeigneter Präparate wird hingegen von der Praxis häufig angegeben.

4. Teilflächenbehandlungen mit Pflanzenschutzmitteln scheinen besonders für das Segment der Herbizide weitestgehend etabliert zu sein. Möglicherweise liegt dies an der guten optischen Erfassbarkeit des Schaderregers, im Falle von nesterartigem Auftreten (z. B. Disteln) sogar direkt bei der Anwendung vom Schlepper aus. Die Messbarkeit bzw. Visualisierbarkeit für den Bereich der teilflächenspezifischen Herbizidapplikation wird dabei auch aus der Landtechnik aufgegriffen (Krebs et al. 2016). Insekten und sporulierende Pilzbestände hingegen sind mobiler bzw. visuell nicht zu erfassen. Eine Teilflächenbekämpfung ist daher bisher technisch noch nicht möglich. Im Raps haben sich nur Teilflächenbehandlungen (Randbehandlungen) gegen Kohlschotenrüssler und Kohlschotenmücke als sinnvoll und praktikabel erwiesen (Furth 2020).

5. Resistenzstrategien können nur eingesetzt werden, wenn durch die Anwender ein Problembewusstsein auftritt. Dieses Problembewusstsein äußerte sich im Rahmen der Befragung über zwei Wege. Zum einen das direkte Wahrnehmen von Bekämpfungsproblemen diverser Schaderreger in verschiedenen Kulturen, zum anderen die Berücksichtigung von Resistenzstrategien in der Präparatauswahl. In der Präparatauswahl zeigt sich, dass Wirksamkeit und Resistenzmanagement die höchsten Einflussfaktoren darstellen. Bevor es also zu Bekämpfungsproblemen durch Resistenzen kommen kann, scheint dem überwiegenden Anteil der Befragten die Problematik und damit das vorbeugende Handeln bewusst zu sein. Dem gegenüber zeigt sich jedoch, dass bereits ein Drittel der befragten Betriebe Probleme durch vermutete bzw. wahrgenommene Resistenz auf seinen Flächen registriert hat.

6. Erfolgskontrollen, gerade unter der zuvor angeführten Resistenzproblematik, sind daher unabdingbar. Überwiegend wird hier auf allen Schlägen für alle drei erfragten Pflanzenschutzmittelgruppen eine Nachkontrolle durchgeführt. Die Schadinsekten werden tendenziell weniger stark kontrolliert; möglicherweise liegt dies daran, dass es Probleme gibt, diese zu erkennen (Abb. 18). Der hohe Anteil an Spritzfenstern, welche zur Kontrolle genutzt werden, scheint auf den ersten Blick unglaubwürdig. Zumal das Spritzfenster unter den zur Erfassung genannten Hilfsmitteln auch nur in der Kategorie „Sonstiges“ auftrat. Es könnte sich jedoch auch um Spritzfenster im wei- 
teren Sinne handeln, etwa Keile oder Spritzfehler, welche zur Kontrolle hinzugezogen werden.

7. Der Einsatz von Prognosemodellen wird auf etwa $20 \%$ der Betriebe als regelmäßig angegeben. Aus einer Studie zur Digitalisierung in der Landwirtschaft geht hervor, dass $93 \%$ der dort Befragten zustimmen, dass die Digitalisierung einen deutlichen Beitrag zum Umweltschutz durch Einsparung von Pflanzenschutz und Düngemitteln leisten kann. Auf insgesamt $82 \%$ der Betriebe werden nach eigenen Angaben Anwendungen der SmartFarming-Technologie eingesetzt (Rohleder et al. 2020). Einem Ausbau der Nutzung von Prognosemodellen ist demnach optimistisch entgegen zu sehen. Der Beratungsbezug wurde zu fast $70 \%$ über die Offizialberatung der Länder angegeben. Interessant ist, dass trotz dieses hohen Bezugs an fachlich fundierter Beratung der Offizialberatung und dem örtlichen Landhandel ein nahezu gleicher Einfluss auf die Entscheidungsfindung im Pflanzenschutz zugeschrieben wird. Möglicherweise läuft die Beratung hier Hand in Hand, sodass die Aussagen der Offizialberatung Eingang in die Beratung durch den lokalen Landhandel finden. Dabei nimmt die Offizialberatung auch Informationen aus der Industrie auf. Andererseits besteht über den Landhandel ein persönlicher, regelmäBiger Kontakt auch der Landwirte, welche keine intensiveren Beratungsvereinbarungen mit der Offizialberatung haben. Hier besteht deutschlandweiter Forschungsbedarf bezüglich des Vergleichs möglicher Beratungsangebote, deren Beratungsausrichtung und die Wirkung auf die Pflanzenschutzentscheidungen auf den Betrieben.

8. Die Anwendung von Schadschwellen scheint innerhalb der befragten Gruppe undifferenziert kritisiert. Insgesamt schätzen $17 \%$ der Befragten die gegebenen Schadschwellen als nicht realistisch ein und sehen sie damit als allgemeines Hemmnis gegen den IPS. Die aus Abschn. 3.8 erkenntlichen Ergebnisse zeigen, dass besonders in den Hauptkulturen Weizen und Mais die Schadschwellen für Pilze und Schadinsekten kritisch betrachtet werden. Inwieweit die geringe Kritik von $6 \%$ an den Schadschwellen für Unkräuter und Gräser an der Gesamtkritik von $17 \%$ beteiligt ist, kann nicht bewertet werden. Möglicherweise wird das Bewusstsein für eine Problematik erst durch konkretes Erfragen gestärkt. So wurde die allgemeine Kritik etwa gestützt erfasst, die übrigen Kritiken an den Schadschwellen hingegen offen abgefragt. Der Einsatz von Hilfsmitteln in der Schaderregerkontrolle ist differenziert $\mathrm{zu}$ betrachten. Für viele Erreger können keine gezielten Hilfsmittel wie Zählrahmen oder Gelbschalen im Feld verwendet werden (z.B. Pilzkrankheiten). Die Nutzung keiner Hilfsmittel bedeutet in diesem Zusammenhang nicht, dass keine Befallskontrollen erfolgen. Es ist davon auszugehen, dass die betriebliche Erfahrung um das regionalspezifische
Aufkommen von Schaderregern ausreichen kann, um eine qualifizierte Erfassung zu gewährleisten. Darüber hinaus werden für Nordrhein-Westfalen aktuell keine Schadschwellen für Unkräuter und Ungräser herausgegeben, welche der Landwirt mit etwa einem Zählrahmen erfassen könnte (Klingenhagen 2020). Dies zeigt die Notwendigkeit der fortlaufenden Evaluierung und Aktualisierung von Schadschwellen insgesamt. Der Einsatz von Gelbschalen ist mit knapp 70\% innerhalb der Gruppe der Rapsanbauer gering, da zur Überwachung und Handlungsentscheidung dieses Instrument als unumgänglich betrachtet wird.

9. Insgesamt scheinen die Hemmnisse bei Zeitaufwand und wahrgenommenem Risiko am höchsten zu liegen. Hier kann davon ausgegangen werden, dass in der Methodensicherheit der integrierten Regulierung bestimmter Schadorganismen offenbar ein Risiko wahrgenommen wird, welches die Umsetzung von Methoden verhindert (vgl. Thiel et al. 2019)

Die durch den NAP angedachten, kulturspezifischen Leitlinien zum integrierten Pflanzenschutz könnten eine Abhilfe leisten, die aufgeführten Punkte zu verbessern und einheitlicher zu betrachten. Aktuell liegen diese für viele Kulturpflanzen noch nicht vor. Exemplarisch seien die Leitlinien für Raps und Mais erwähnt, welche durch den wissenschaftlichen Beirat bereits begutachtet wurden und daher der Öffentlichkeit zur Verfügung stehen (LfL et al. 2019; Bartels et al. 2020).

Insgesamt zeichnet sich ein Bild des IPS, welches eine bisher unvollständige Umsetzung darstellt. Dies hängt an zweierlei Faktoren:

1. Einige Vorgaben sind widersprüchlich, betrachtet man etwa die Bodenbearbeitung. Unter optimalen Witterungsbedingungen kann der Pflugeinsatz einen Beitrag zum IPS leisten. Direktsaatsysteme basieren bisher hingegen häufig auf Totalherbiziden und Systeme mit permanenter Cover-Crop oder Mulchsysteme zur Unkrautunterdrückung und stehen auch aufgrund der umfassenden Einsatzmöglichkeiten von Glyphosat erst am Anfang. An anderer Stelle fehlen dem Anwender die Möglichkeiten, rückt man etwa die Anwendung von Schadschwellen in den Blickpunkt. Wenn keine regionalspezifischen Schadschwellen, etwa für Ungräser, herausgegeben werden, kann auch nicht nach ihnen gehandelt werden.

2. Einzelne der acht Grundsätze werden nicht im ausreichenden Maß vollumfänglich umgesetzt, obwohl die Vorgaben und Methoden nicht widersprüchlich und prinzipiell gut anwendbar sind. Dazu zählen etwa Feldkontrollen nach definierten Mustern (z.B. Linienbonitur) oder der Einsatz mechanischer Beikrautregulierung. Letzterer ist dabei selbstverständlich immer an die In- 
vestition entsprechender Geräte durch die Betriebe geknüpft, jedoch scheint die Bereitschaft, diese Techniken zu testen, nicht ausgeprägt zu sein.

Gleichwohl ist zu beachten, dass es sich bei dieser Studie um eine Näherung über verschiedene Parameter handelt, welche nicht unabhängig und nach objektiven Kriterien gemessen oder beobachtet wurde. Viel mehr waren es Selbstauskünfte landwirtschaftlicher Betriebe, welche somit auch ihre Auffassungen und Sichtweisen widergeben.

\section{Schlussfolgerung}

Trotz einer langjährigen Verankerung im Pflanzenschutzgesetz und der ausführlichen Handreichung der acht allgemeinen Grundsätze des integrierten Pflanzenschutzes werden viele Aspekte des integrierten Pflanzenschutzes in der Praxis nicht umgesetzt. Aspekte zur Resistenzvermeidung, der Zielartenspezifität von Pflanzenschutzmitteln, Erfolgskontrollen der Pflanzenschutzmaßnahmen sowie Reduzierung der Aufwandmengen über Teilflächenapplikationen haben sich im praktisch umsetzbaren Rahmen auf den Betrieben aber etabliert. Gerade im Bereich der Schadschwellennutzung sowie in den einzelnen Aspekten der Vorbeugung von Schadorganismen gibt es aus landwirtschaftlicher Sicht Widersprüche bzw. für die Praxis fehlende Handreichungen, welche eine umfangreichere Umsetzung des IPS erschweren. Gleichwohl sind im Bereich der Feldkontrollen sowie der nicht-chemischen Methoden (speziell Beikrautregulierung) noch deutliche Umsetzungslücken seitens der Praxis festzustellen. Die nicht ausreichend umgesetzten Grundsätze sowie die angeführten Kritikpunkte bedürfen einer Überprüfung bzw. Überarbeitung durch Wissenschaft und Praxis, bei der auch zu prüfen ist, inwiefern wirtschaftliche Fehlanreize oder ungeeignete Regulierungen eine mangelnde Umsetzung begründen.

Danksagung Die Daten stammen aus dem Projekt „Aktueller Umsetzungsstatus des integrierten Pflanzenschutzes in Nordwest-Deutschland“ (IPS-Nordwest). Das Projekt startete im September 2019 und endete im März 2020. An dieser Stelle sei den landwirtschaftlichen Betrieben gedankt, welche sich zur Teilnahme bereiterklärten.

Förderung Das Projekt wurde gefördert durch das Ministerium für Landwirtschaft, Natur- und Verbraucherschutz (MULNV) des Landes Nordrhein-Westfalen und an der Fachhochschule Südwestfalen, Standort Soest, bearbeitet.

Funding Open Access funding enabled and organized by Projekt DEAL.

Interessenkonflikt L. Thiel, M. Mergenthaler und V. Haberlah-Korr geben an, dass kein Interessenkonflikt besteht.

Open Access Dieser Artikel wird unter der Creative Commons Namensnennung 4.0 International Lizenz veröffentlicht, welche die Nut- zung, Vervielfältigung, Bearbeitung, Verbreitung und Wiedergabe in jeglichem Medium und Format erlaubt, sofern Sie den/die ursprünglichen Autor(en) und die Quelle ordnungsgemäß nennen, einen Link zur Creative Commons Lizenz beifügen und angeben, ob Änderungen vorgenommen wurden.

Die in diesem Artikel enthaltenen Bilder und sonstiges Drittmaterial unterliegen ebenfalls der genannten Creative Commons Lizenz, sofern sich aus der Abbildungslegende nichts anderes ergibt. Sofern das betreffende Material nicht unter der genannten Creative Commons Lizenz steht und die betreffende Handlung nicht nach gesetzlichen Vorschriften erlaubt ist, ist für die oben aufgeführten Weiterverwendungen des Materials die Einwilligung des jeweiligen Rechteinhabers einzuholen.

Weitere Details zur Lizenz entnehmen Sie bitte der Lizenzinformation auf http://creativecommons.org/licenses/by/4.0/deed.de.

\section{Literatur}

Bartels A, Haberlah-Korr V, Schäfer BC (2020) Leitlinie des integrierten Pflanzenschutzes im Rapsanbau. Nationaler Aktionsplan Pflanzenschutz. https://www.nap-pflanzenschutz.de/fileadmin/ SITE_MASTER/content/IPS/Integrierter_Pflanzenschutz/ Leitlinien_IPS/201111_RL_UFOP_1738_Leitlinie_Raps_final. pdf. Zugegriffen: 15. Dez. 2020

BayWa (2020) E-Pura, elektronische Saatgutbeize. http://www.e-pura. de/vorteile.html. Zugegriffen: 7. Juli 2020

Bundesministerium für Ernährung und Landwirtschaft (2020) Grundsätze des integrierten Pflanzenschutzes. https://www.nappflanzenschutz.de/praxis/integrierter-pflanzenschutz/grundsaetzeips/. Zugegriffen: 15. Juni 2020

Bundesministerium für Ernährung, Landwirtschaft und Verbraucherschutz (2010) Gute fachliche Praxis im Pflanzenschutz. https:// www.bmel.de/SharedDocs/Downloads/Broschueren/GutePraxis Pflanzenschutz.pdf?_blob=publicationFile. Zugegriffen: 1. März 2019

Bundesminsiterium für Ernährung und Landwirtschaft (2017) Nationaler Aktionsplan zur nachhaltigen Anwendung von Pflanzenschutzmitteln. BMEL, Bonn

Bundessortenamt (2019a) Beschreibende Sortenliste Getreide, Mais, Öl- und Faserpflanzen, Leguminosen, Rüben, Zwischenfrüchte. Bundessortenamt, Hannover

Bundessortenamt (2019b) Beschreibende Sortenliste Kartoffeln. Bundessortenamt, Hannover

Diekmann A (2014) Empirische Sozialforschung. Grundlagen, Methoden, Anwendungen, 9. Aufl. Rowohlt, Reinbek bei Hamburg

Esch F-R (2021) Top of mind. Gabler Wirtschaftslexikon, Springer Verlag. https://wirtschaftslexikon.gabler.de/definition/top-mind47323/version-270589. Zugegriffen: 16. Febr. 2021

Estler M (2011) Lehrbuch des Pflanzenbaues. In: Lütke Entrup N, Schäfer BC (Hrsg) Kulturpflanzen, 3. Aufl. Bd. 2. AgroConcept, Bonn

Furth U (2020) Schädlinge im Raps. In: Paffrath P, Schauhoff-Tholen V, Röhling D (Hrsg) Ratgeber Pflanzenbau und Pflanzenschutz. Landwirtschaftskammer Nordrhein-Westfalen, Köln

Haller S, Moakes S, Niggli U, Riedel J, Stolze M, Thompson M (2020) Entwicklungsperspektiven der ökologischen Landwirtschaft in Deutschland. Umweltbundesamt, Dessau-Roßlau

Hokkanen H-M (2015) Integrated pest management at the crossroads: science, politics or business (as usual)? Arthropod Plant Interact 9:543-545

Jorgensen L, Nielsen G, Jensen J, Orum J, Pinnschmidt H (2008a) Problems with disseminating information on disease control in wheat and barley to farmers. Eur J Plant Pathol 121:303-312 
Jorgensen L, Noe E, Nielsen G, Orum J, Jensen J, Pinnschmidt H (2008b) Integrating disease control in winter wheat - optimizing fungicide input. Outlook on Pest Management, S 206-213

Klingenhagen G (2020) Unkräuter in Getreide, Mais und Raps. In: Paffrath P, Schauhoff-Tholen V, Röhling D (Hrsg) Ratgeber Pflanzenbau und Pflanzenschutz. Landwirtschaftskammer NordrheinWestfalen, Köln

Krebs M, Rautmann D, Nordmeyer H (2016) Situationsgerechte Unkrautbekämpfung durch Direkteinspeisung von Pflanzenschutzmitteln. Julius Kühn-Institut, Braunschweig https://doi.org/10. 5073/jka.2016.452.032

Kuehne G, Llewellyn R, Panell D, Wilkonson R, Dollig P, Ouzman J, Ewing M (2017) Predicting farmer uptake of new agricultural practices: A tool for research, extension and policy. Agric Syst 156:115-125

Landesanstalt für Landwirtschaft, Deutsches MaisKomitee, Landwirtschaftliches Technologiezentrum Augustenberg, Landwirtschaftskammer Niedersachsen (2019) Leitlinien zum integrierten Pflanzenschutz im Mais. Deutsches Maiskomitee, Bonn. https:// www.maiskomitee.de/web/upload/pdf/produktion/Leitlinie_IPS_ Mais_2019.pdf. Zugegriffen: 31. Juli 2020

Landwirtschaftskammer Nordrhein-Westfalen (2017) Zahlen zur Landwirtschaft in Nordrhein-Westfalen 2017. Fachbereich 51 der Landwirtschaftskammer Nordrhein-Westfalen, Münster

Limagrain (2021) Wurzelhals- und Stängelfäule im Raps. https://www. lgseeds.de/raps/phoma/ (Beispiel als Werbung für Sortenmerkmale, welche nicht in der BSL aufgeführt sind). Zugegriffen: 6. Jan. 2021

Llewellyn R, Lindner R, Panell D, Powels S (2007) Herbicide resistance and the adoption of integratet weed management by western Australian grain growers. Agric Econ 36:123-130

Müller C (2001) Die gute fachliche Praxis im Pflanzenschutz-, Düngemittel- und Bodenschutzrecht - Ausprägungen auf die landwirtschaftliche Bodennutzung, 1. Aufl. Verlag Dr. Kovač, Hamburg

Niedersächsisches Ministerium für Ernährung, Landwirtschaft und Verbraucherschutz (2020) Die niedersächsische Landwirtschaft in Zahlen (2017). https://www.ml.niedersachsen.de/startseite/ aktuelles_veranstaltungen/veroeffentlichungen/dieniedersaechsische-landwirtschaft-in-zahlen-121348.html. Zugegriffen: 5. Jan. 2021
Rohleder B, Krüsken B, Reinhardt H (2020) Digitalisierung in der Landwirtschaft 2020. Repräsentative Befragung des Deutschen Bauernverbandes, der landwirtschaftlichen Rentenbank und des Digitalverbands Bitkom. https://www.bauernverband. de/fileadmin/user_upload/dbv/pressemitteilungen/2020/KW_18/ 200427_PraesentationPKLW_final.pdf. Zugegriffen: 8. Juli 2020

Statistisches Amt für Hamburg und Schleswig-Holstein (2017) Die Bodennutzung in Schleswig-Holstein 2016. https://www.statistiknord.de/fileadmin/Dokumente/Statistische_Berichte/ landwirtschaft/C_IV_Teil_1_S_Bodennutzung_Agrarstruktur/ C_IV_ASE2016_Teil_1_SH_korr.pdf. Zugegriffen: 5. Jan. 2021

Statistisches Amt für Hamburg und Schleswig-Holstein (2019) Betriebsverhältnisse in den landwirtschaftlichen Betrieben Schleswig-Holsteins 2016. https://www.statistik-nord.de/fileadmin/ Dokumente/Statistische_Berichte/landwirtschaft/C_IV_Teil_7_ S_Betriebe/C_IV_ASE2016_Teil_7_SH.pdf. Zugegriffen: 5. Jan. 2021

Statistisches Bundesamt (2017) Land- und Forstwirtschaft, Fischerei. Arbeitskräfte und Berufsbildung der Betriebsleiter/Geschäftsführer. Agrarstrukturerhebung 2016

Thiel L, Haberlah-Korr V, Gerowitt B, Mergenthaler M (2019) Ich würde wohl das meiste Geld mit Spaziergängen über den Acker verdienen - Die Sicht von Betriebsleitenden zum Einsatz von Insektiziden im integrierten Pflanzenschutz. Berichte über Landwirtschaft, S 97-93

Zeng W, Wang D, Kirk W, Hao J (2012) Use of Coniothyrium minitans and other microorganisms for reducing Sclerotinia sclerotiorum. Biol Control 60(2):225-232. https://doi.org/10.1016/j.biocontrol. 2011.10.009

Lukas Thiel $(* 02.11 .1994)$ aus Kamen stammt von einem landwirtschaftlichen Marktfruchtbetrieb der Hellwegbörde. Nach dem Abitur 2013 begann er ein Bachelorstudium der Agrarwirtschaft an der Fachhochschule in Soest. Nach Abschluss des Bachelorstudiums folge ein Masterstudium der Agrarwirtschaft, ebenfalls in Soest. Seit 2018 ist Lukas Thiel wiss. Mitarbeiter des Fachbereichs Agrarwirtschaft an der Fachhochschule Südwestfalen mit Schwerpunkt Pflanzenschutz. 